\title{
Perturbation Theory for Particle in a Box
}

\author{
H. Kleinert, ${ }^{*}$ A. Chervyakov ${ }^{\dagger}$, and B. Hamprecht \\ Institut für Theoretische Physik \\ Freie Universität Berlin \\ Arnimallee14, D-14195 Berlin
}

\begin{abstract}
Recently developed strong-coupling theory open up the possibility of treating quantum-mechanical systems with hard-wall potentials via perturbation theory. To test the power of this theory we study here the exactly solvable quantum mechanics of a point particle in a one-dimensional box. Introducing an auxiliary harmonic mass term $m$, the groundstate energy $E^{(0)}$ can be expanded perturbatively in powers of $1 / m d$, where $d$ is the box size. The removal of the infrared cutoff $m$ requires the resummation of the series at infinitely strong coupling. We show that strong-coupling theory yields a fast-convergent sequence of approximations to the well-known quantum-mechanical energy $E^{(0)}=\pi^{2} / 2 d^{2}$.
\end{abstract}

1. Variational perturbation theory [1] permits us to convert divergent weak-coupling expansions into convergent strong-coupling expansions. In particular, a constant strong-coupling limit of a function can be evaluated from its weak-coupling expansion with any desired accuracy. As an important application, this has led to a novel way of calculating critical exponents without using the renormalization group [2].

\footnotetext{
*E-mail: kleinert@physik.fu-berlin.de

${ }^{\dagger}$ On leave from LCTA, JINR, Dubna, Russia, E-mail: chervyak@physik.fu-berlin.de
}

Given this theory, new classes of physical systems become accessible to perturbation theory. For instance, the important problem a the pressure exerted by a stack of membranes upon the enclosing walls [3] has now become calculable analytically with the help of perturbation theory. For a single membrane, this has already be done successfully [4]5]. Realistic physical problems have usually the disadvantage that the maximally accessible order of perturbation theory is quite limited. If we want to gain a better understanding of the convergence of the successive approximations as the order goes to infinity it is useful to study a system where the result is known exactly. This will be done in the present note for a quantum-mechanical point particle in a one-dimensional box. The ground state energy of this system is known exactly, $E^{(0)}=\pi^{2} / 2 d^{2}$ (in natural units), where $d$ is size the of the box. We shall demonstrate how this result is found via strong-coupling theory from a perturbation expansion, thus illustrating the reliability of the earlier membrane calculations [4.5].

2. The partition function of a particle in a box is given by the euclidean path integral (always in natural units)

$$
Z=\int \mathcal{D} u(t) e^{\frac{1}{2} \int d t(\partial u)^{2}}
$$


where the particle coordinate $u(t)$ is restricted to the interval $-d / 2 \leq u(t) \leq d / 2$. Since such a hard-wall restriction is difficult to treat analytically in the path integral (11), we make the hard-walls soft by adding to the euclidean action $E$ in the exponent of (11) a potential term diverging near the walls. Thus we consider the auxiliary euclidean action

$$
E=\frac{1}{2} \int d t\left\{[\partial u(t)]^{2}+V(u(t))\right\}
$$

where $V(u)$ is given by

$V(u)=\frac{\omega^{2}}{2}\left(\frac{d}{\pi} \tan \frac{\pi u}{d}\right)^{2}=\frac{\omega^{2}}{2}\left(u^{2}+\frac{2}{3} g u^{4}+\ldots\right)$.

On the right-hand side we have introduced a parameter $g \equiv \pi^{2} / d^{2}$

3. The expansion of the potential in powers of $g$ can now be treated perturbatively, leading to an expansion of $Z$ around the harmonic part of the partition function, in which the integrations over $u(t)$ run over the entire $u$-axis and yield

$$
Z_{\omega}=e^{-(1 / 2) \operatorname{Tr} \log \left(\partial^{2}+\omega^{2}\right)}
$$

For $L \rightarrow \infty$, the exponent gives a free energy density $f=-L^{-1} \log Z$ equal to the ground state energy of the harmonic oscillator

$$
f_{0}=\frac{\omega}{2}
$$

The treatment of the interaction terms can be organized in powers of $g$, and give rise to an expansion of the free energy with the generic form

$$
f=f_{0}+\omega \sum_{k=1}^{\infty} a_{k}\left(\frac{g}{\omega}\right)^{k}
$$

The calculation of the coefficients $a_{k}$ in this expansion proceeds as follows. First we expand the potential in (2) to identify the power series for the interaction energy

$$
\begin{aligned}
E^{\text {int }} & =\frac{\omega^{2}}{2} \int d t\left\{g \varepsilon_{4} u^{4}+g^{2} \varepsilon_{6} u^{6}+g^{3} \varepsilon_{8} u^{8}+\ldots\right\} \\
& =\frac{\omega^{2}}{2} \sum_{k=1}^{\infty} \int d t g^{k} \varepsilon_{2 k+2}\left[u^{2}(t)\right]^{k+1},
\end{aligned}
$$

with coefficients

$$
\begin{aligned}
& \varepsilon_{4}=\frac{2}{3}, \quad \varepsilon_{6}=\frac{17}{45}, \quad \varepsilon_{8}=\frac{62}{315}, \quad \varepsilon_{10}=\frac{1382}{14175}, \\
& \varepsilon_{12}=\frac{21844}{467775}, \quad \varepsilon_{14}=\frac{929569}{42567525}, \quad \varepsilon_{16}=\frac{6404582}{638512875}, \\
& \varepsilon_{18}=\frac{443861162}{97692469875}, \quad \varepsilon_{20}=\frac{18888466084}{9280784638125}, \\
& \varepsilon_{22}=\frac{113927491862}{126109485376875}, \quad \varepsilon_{24}=\frac{58870668456604}{147926426347074375}, \\
& \varepsilon_{26}=\frac{8374643517010684}{48076088562799171875}, \\
& \varepsilon_{28}=\frac{689005380505609448}{9086380738369043484375}, \\
& \varepsilon_{30}=\frac{129848163681107301953}{3952575621190533915703125}, \\
& \varepsilon_{32}=\frac{1736640792209901647222}{122529844256906551386796875}, \\
& \varepsilon_{34}=\frac{418781231495293038913922}{68739242628124575327993046875}, \ldots
\end{aligned}
$$

The interaction terms $\int d t\left[u^{2}(t)\right]^{k+1}$ and their products are expanded according to Wick's rule into sums of products of Wick contractions representing harmonic twopoint correlation functions

$$
\left\langle u\left(t_{1}\right) u\left(t_{2}\right)\right\rangle=\int \frac{d k}{2 \pi} \frac{e^{i k\left(t_{1}-t_{2}\right)}}{k^{2}+\omega^{2}}=\frac{e^{-\omega\left|t_{1}-t_{2}\right|}}{2 \omega} .
$$

Associated local expectation values are $\left\langle u^{2}\right\rangle=1 / 2 \omega$, and

$$
\begin{aligned}
\langle u \partial u\rangle & =\int \frac{d k}{2 \pi} \frac{k}{k^{2}+\omega^{2}}=0 \\
\langle\partial u \partial u\rangle & =\int \frac{d k}{2 \pi} \frac{k^{2}}{k^{2}+\omega^{2}}=-\frac{\omega}{2}
\end{aligned}
$$

where the last integral is calculated using dimensional regularization in which $\int d k k^{\alpha}=0$ for all $\alpha$. The Wick 
contractions are organized with the help of Feynman diagrams. Only the connected diagrams contribute to the free energy density. The graphical expansion of free energy up to four loops is

$$
\begin{aligned}
& f=\frac{\omega}{2}+\left(\frac{\omega^{2}}{2}\right)\left\{g \varepsilon_{4} 3 \bigcirc+g^{2} \varepsilon_{6} 15 \oslash+g^{3} \varepsilon_{8} 105 \bigotimes\right\} \\
& -\frac{1}{2 !}\left(\frac{\omega^{2}}{2}\right)^{2}\left\{g^{2} \varepsilon_{4}^{2}[72 \infty 00+24 \Theta]\right. \\
& \left.+g^{3} 2 \varepsilon_{4} \varepsilon_{6}[540 \oslash \infty+360 \bigcirc 0]\right\} \\
& +\frac{1}{3 !}\left(\frac{\omega^{2}}{2}\right)^{3} g^{3} \varepsilon_{4}^{3}\{25920000+1728 \text { ठ? } \\
& +3456 \oslash 0+1728 \varnothing\} \text {. }
\end{aligned}
$$

Note different numbers of loops contribute to the terms of order $g^{n}$. The calculation of the diagrams in Eq. (11) is simplified by the factorization property: If a diagram consists of two subdiagrams touching each other at a single vertex, the associated Feynman integral factorizes into those of the subdiagrams. In each diagram, the last $t$-integral yields an overall factor $L$, due to translational invariance along the $t$-axis, the others produce a factor $1 / \omega$. Using the explicit expression (10) for the lines in the diagrams, we find the following velues for the Feynman integrals:

$$
\begin{aligned}
& \infty=L \frac{1}{16 \omega^{5}}, \quad \infty 00=L \frac{1}{64 \omega^{8}}, \\
& \ominus=L \frac{1}{32 \omega^{5}}, \quad \text { ठ }=L \frac{3}{128 \omega^{8}}, \\
& \varnothing 0=L \frac{1}{32 \omega^{6}}, \quad छ=L \frac{5}{8 \cdot 64 \cdot \omega^{8}}, \\
& \oslash=L \frac{1}{32 \omega^{6}}, \quad \varnothing=L \frac{3}{8 \cdot 64 \cdot \omega^{8}}
\end{aligned}
$$

Adding all contributions in (11), we obtain up to the order $g^{3}$ :

$$
\begin{aligned}
f_{3}= & \omega\left\{\frac{1}{2}+\frac{3}{8} \varepsilon_{4}\left(\frac{g}{\omega}\right)+\left[\frac{15}{16} \varepsilon_{6}-\frac{21}{32} \varepsilon_{4}^{2}\right]\left(\frac{g}{\omega}\right)^{2}+\right. \\
& \left.+\left[\frac{105}{32} \varepsilon_{8}-\frac{45}{8} \varepsilon_{4} \varepsilon_{6}+\frac{333}{128} \varepsilon_{4}^{3}\right]\left(\frac{g}{\omega}\right)^{3}\right\},
\end{aligned}
$$

which has the generic form (6). We can go to higher orders by extending the Bender-Wu recursion relation for the ground-state energy of the quartic anharmonic oscillator as follows:

$$
\begin{aligned}
& 2 j C_{n, j}=(j+1)(2 j+1) C_{n, j}- \\
& \frac{1}{2} \sum_{k=1}^{n}(-1)^{k} \varepsilon_{2 k+2} C_{n-k}, j-k-1 \\
& -\sum_{k=1}^{n-1} C_{k, 1} C_{n-k}, j, \quad 1 \leq j \leq 2 n, \\
& C_{0,0}=1, \quad C_{n, j}=0 \quad(n \geq 1, j<1) .
\end{aligned}
$$

After solving these recursion relations, the coefficients $a_{k}$ in (6) are given by $a_{k}=(-1)^{k+1} C_{k, 1}$. For brevity, we list here the first sixteen expansion coefficients for $f$, calculated with the help of the algebra program REDUCE:

$$
\begin{aligned}
& a_{0}=\frac{1}{2}, a_{1}=\frac{1}{4}, a_{2}=\frac{1}{16}, a_{3}=0, a_{4}=-\frac{1}{256}, a_{5}=0, \\
& a_{6}=\frac{1}{2048}, a_{7}=0, a_{8}=-\frac{5}{65536}, a_{9}=0, \\
& a_{10}=\frac{7}{524288}, a_{11}=0, a_{12}=-\frac{21}{8388608}, a_{13}=0, \\
& a_{14}=\frac{33}{67108864}, a_{15}=0, a_{16}=-\frac{429}{4294967296}, \ldots .
\end{aligned}
$$

4. We are now ready to calculate successive strongcoupling approximations to the function $g(g)$ and suty the convergence behavior as the order grows large. According to the general theory in [2,1], the $N$ th order approximation to the strong-coupling limit $f^{*}$ of the series $f(g)$ is found by replacing, in the truncated series after the $N$ th term $f_{N}(g)$, the frequency $\omega$ by the identical expression $\sqrt{\Omega^{2}+g r}$, where $r \equiv\left(\omega^{2}-\Omega^{2}\right) / g$ which is, 
however, treated for a moment as an independent variable, whereas $\Omega$ is a dummy parameter. Then the square root is expanded binomially in powers of $g$, and $f_{N}(g)$ is reexpanded up to order $g^{N}$. After that, $r$ is replaced by its proper value. In this way we obtain a function $f_{N}(g, \Omega)$ which depends on $\Omega$, which thus becomes a variational parameter. The best approximation is obtained by extremizing $f_{N}(g, \Omega)$ with respect to $\omega$. The strongcoupling limit is obtained by taking $g$ to infinity which is equivalent to setting $\omega=0$. In this limit, the optimal $\Omega$ will grow proportionally with $g$, so that $g / \Omega=1 / c$ is finite, and the variational expression $f_{N}(g, \Omega)$ becomes a function of $f_{N}(c)$. For $\omega=0$, the above reexpansion amounts simply to replacing each power $\omega^{n}$ in each expansion terms of $f_{N}(g)$ by the binomial expansion of $(1-1)^{-n / 2}$ truncated after the $(N-n)$ th term. The first nine variational functions $f_{N}(c)$ are listed in Table 1.

TABLE I. First eight variational functions $f_{N}(c)$.

$$
\begin{aligned}
& f_{2}(c)=\frac{1}{4}+\frac{1}{16 c}+\frac{3 c}{16} \\
& f_{3}(c)=\frac{1}{4}+\frac{3}{32 c}+\frac{5 c}{32} \\
& f_{4}(c)=\frac{1}{4}-\frac{1}{256 c^{3}}+\frac{15}{128 c}+\frac{35 c}{256} \\
& f_{5}(c)=\frac{1}{4}-\frac{5}{512 c^{3}}+\frac{35}{256 c}+\frac{63 c}{512} \\
& f_{6}(c)=\frac{1}{4}+\frac{1}{2048 c^{5}}-\frac{35}{2048 c^{3}}+\frac{315}{2048 c}+\frac{231 c}{2048} \\
& f_{7}(c)=\frac{1}{4}+\frac{7}{4096 c^{5}}-\frac{105}{4096 c^{3}}+\frac{693}{4096 c}+\frac{429 c}{4096} \\
& f_{8}(c)=\frac{1}{4}-\frac{5}{65536 c^{7}}+\frac{63}{16384 c^{5}}-\frac{1155}{32768 c^{3}}+\frac{3003}{16384 c}+\frac{6435 c}{65536} \\
& f_{9}(c)=\frac{1}{4}-\frac{45}{131072 c^{7}}+\frac{231}{32768 c^{5}}-\frac{3003}{65536 c^{3}}+\frac{6435}{32768 c}+\frac{12155 c}{131072} \\
& \hline
\end{aligned}
$$

The functions $f_{N}(c)$ are minimized starting from $f_{2}(c)$ and searching the minimum of each successive $f_{3}(c)$, $f_{3}(c), \ldots$ nearest to the previous one. The functions $f_{N}(c)$ together with their minima are plotted in Fig. 11.

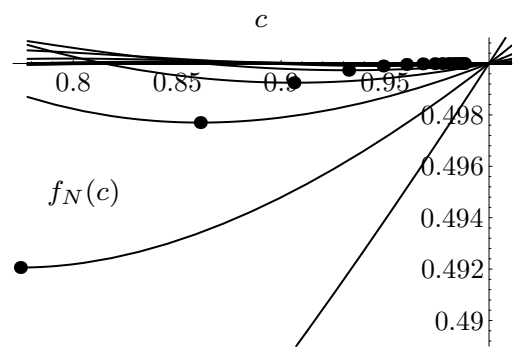

FIG. 1. Variational functions $f_{N}(c)$ up to $N=16$ are shown together with their minima whose $y$-coordinates approach rapidly the correct limiting value $1 / 2$.

The minima lie at

$$
\begin{aligned}
\left(N, f_{N}^{\min }\right)= & (2,0.466506),(3,0.492061),(4,0.497701), \\
& (5,0.499253),(6,0.499738),(7,0.499903), \\
& (8,0.499963),(9,0.499985),(10,0.499994), \\
& (11,0.499998),(12,0.499999),(13,0.5000), \\
& (14,0.50000),(15,0.50000),(16,0.5000) .(16)
\end{aligned}
$$

They converge exponentially fast against the known result 1/2, as shown in Fig. 2.

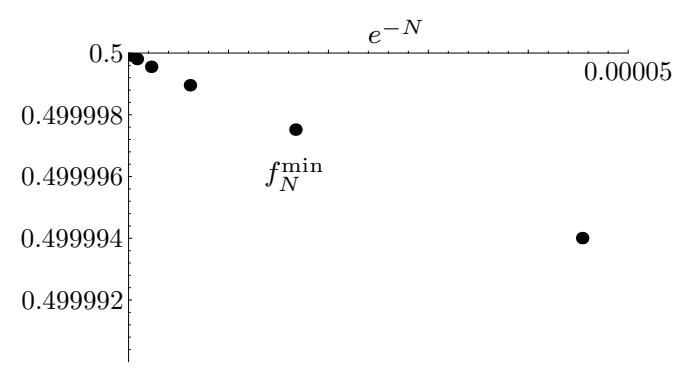

FIG. 2.

5. The alert reader will have noted that the expansion 
coefficients (15) possesses two special properties: First, they lack the factorial growth at large orders which would be found for a single power $\left[u^{2}(t)\right]^{k+1}$ of the interaction potential [6]. The factorial growth is canceled by the specific combination of the different powers in the interaction (7), making the series (6) convergent inside a certain circle. Still, since this circle has a finite radius (the ratio test shows that it is unity), this convergent series cannot be evaluated in the limit of large $g$ which we want to do, so that variational strong-coupling theory is not superfluous. However, there is a second remarkable property of the coefficients (15): They contain an infinite number of zeros in the sequence of coefficients for each odd number, except for the first one. We may take advantage of this property by separating off the irregular term $a_{1} g=g / 4=\pi^{2} / 4 d^{2}$, setting $\alpha=g^{2} / 4 \omega^{2}$, and rewriting $f$ as

$f=\frac{\pi^{2}}{4 d^{2}}\left[1+\frac{1}{\sqrt{\alpha}} h(\alpha)\right], \quad h(\alpha) \equiv \sum_{n=0}^{N} 2^{2 n+1} a_{2 n} \alpha^{n}$.

Inserting the numbers (15), the expansion of $h(\alpha)$ reads

$$
\begin{aligned}
h(\alpha)=1+\frac{\alpha}{2} & -\frac{\alpha^{2}}{8}+\frac{\alpha^{3}}{16}-\frac{5}{128} \alpha^{4}+\frac{7}{256} \alpha^{5}- \\
& -\frac{21}{1024} \alpha^{6}+\frac{33}{2048} \alpha^{7}-\frac{429}{32768} \alpha^{8}+\ldots
\end{aligned}
$$

We now realize that this is the bibomial power series expansion of $\sqrt{1+\alpha}$. Substituting this into (17), we find the exact ground state energy for the euclidean action (2)

$$
E^{(0)}=\frac{\pi^{2}}{4 d^{2}}\left(1+\sqrt{1+\frac{1}{\alpha}}\right)=\frac{\pi^{2}}{4 d^{2}}\left(1+\sqrt{1+4 \omega^{2} \frac{d^{4}}{\pi^{4}}}\right)
$$

Here we can go directly to the strong-coupling limit $\alpha \rightarrow$ $\infty$ corresponding to $d \rightarrow 0$, where we recover the exact ground-state energy $E^{(0)}=\pi^{2} / 2 d^{2}$.

5. The energy (19) can of course be obtained directly by solving the Schrödinger equation associated with the potential (7),

$$
\frac{1}{2}\left\{-\frac{\partial^{2}}{\partial x^{2}}+\left[\frac{\lambda(1-\lambda)}{\cos ^{2} x}-1\right]\right\} \psi(x)=\frac{d^{2}}{\pi^{2}} E \psi(x),
$$

where we have replaced $u \rightarrow d x / \pi$ and set $\omega^{2} d^{4} / \pi^{4} \equiv$ $\lambda(\lambda-1)$, so that

$$
\lambda=\frac{1}{2}\left(1+\sqrt{1+4 \omega^{2} \frac{d^{4}}{\pi^{4}}}\right) .
$$

Equation (20) is of the Pöschl-Teller type and has the ground state wave function [7]

$$
\psi_{0}(x)=\text { const } \times \cos ^{\lambda} x
$$

with the eigenvalue $\pi^{2} E^{(0)} / d^{2}=\left(\lambda^{2}-1\right) / 2$, which agrees of xourse with Eq. (19).

If we were to apply the variational procedure to the series $h(\alpha) / \sqrt{\alpha}$ in $f$ of Eq. (19), by replacing the factor $1 / \omega^{2 n}$ contained in each power $\alpha^{n}$ by $\Omega=\sqrt{\Omega^{2}+r \alpha}$ and reexpanding now in powers of $\alpha$ rather than $g$, we would find that all approximation $h_{N}(c)$ would posses a minimum with unit value, such that the corresponding extremal functions $f_{N}(c)$ yield the correct final energy in each order $N$. 
[1] H. Kleinert, Path Integrals in Quantum Mechanics, Statistics and Polymer Physics, World Scientific, Singapore, 1995.

[2] H. Kleinert, Phys. Rev. D 57, 2264 (1998) (APS E-Print aps1997jun25_001); Adendum: ibid. D 58, 1077 (1998) cond-mat/9803268; Strong-Coupling $\phi^{4}$-Theory in $4-\epsilon$ Dimensions and Critical Exponents, cond-mat/9801167); Seven Loop Critical Exponents from Strong-Coupling $\phi^{4}$ Theory in Three Dimensions, Berlin preprint 1998 (hepth/9812197).

[3] W. Helfrich, Z. Naturforsch. A 33, 305 (1978), W. Helfrich, R.M. Servuss, Nuovo Cim. D 3, 137 (1984);

W. Janke, H. Kleinert, Phys. Lett. 58, 144 (1987), W. Janke, H. Kleinert and H. Meinhardt, Phys. Lett. B 217, 525 (1989);

G. Gompper and D.M. Kroll, Europhys. Lett. 9, 58 (1989), R.R. Netz and R. Lipowski, Europhys. Lett. 29. 345 (1995), F. David, J. de Phys. 51, C7-115 (1990).

[4] H. Kleinert, Pressure of Membrane between Walls, Berlin preprint, 1998 cond-mat/9811308.

[5] M. Bachmann, H. Kleinert, and A. Pelster, StrongCoupling Calculation of the Fluctuation Pressure of a Membrane Between Walls, Berlin preprint 1999 condmat/9905397).

[6] C.M. Bender and T.T. Wu, Phys. Rev. 184, 1231 (1969); Phys. Rev. D 7, 1620 (1973).

[7] S. Flügge, Practical Quantum Mechanics I, SpringerVerlag, Berlin-Heidelberg-New York, 1971.
[8] H. Kleinert, Phys. Rev. D 57, 2264 (1998) (www.physik.fu-berlin.de/ ${ }^{\text {kleinert } / 257) ; ~}$ Phys. Lett. B 434, 74 (1998). 